\title{
Non-Profits Vs For-Profits in the Game of Social Media: Critical Observations and Reflections
}

\author{
Michelle Schmude $^{* 1}$ and Scott J. Weiland ${ }^{1}$
}

Department of Mass Communications, King's College, USA

\begin{abstract}
With the goal of understanding social media habits, a panel discussion of almost 100 non-profit and for-profit organizations at "NEPABlogCon" identified similarities and differences in the areas of social media platforms, social media strategic plans, and social media policies between non-profits and for-profits. More social media similarities than differences existed among participants. In addition there are several key areas that should be investigated by participants to mirror best practices.
\end{abstract}

Keywords: Social media; Social media strategic plans; Social media policies; Non-profits; For-profits; Facebook; Twitter; LinkedIn; YouTube; Vimeo; Google+

\section{Introduction}

Understanding why an organization dives into the deep waters of social media can be a complicated and thought provoking matter. To this end, an opportunity to interact with almost 100 non-profit and for-profit organizations to talk social media via a panel discussion was presented and graciously accepted. The goal of the interaction was to understand social media habits. This interaction occurred during a panel discussion at "NEPABlogCon," a gathering of social media junkies and bloggers from around the northeast corner of Pennsylvania, USA. The purpose of the panel discussion was to identify similarities and differences in the areas of social media platforms, social media strategic plans, and social media policies between non-profits and for-profits. It was revealed that more social media similarities than differences existed among participants and that there are several key areas that should be investigated by participants to mirror best practices.

\section{Social media platforms}

At the onset of the panel the group was polled and it was determined that there were an almost equal number of non-profit and for-profit firms represented. Each participant noted that they had a presence on Facebook and Twitter, but there was sporadic and minimal participation among many other tools including LinkedIn, Four Square, and Google+. One key reflection of this exercise illuminated the focus on Facebook and Twitter without logic or reason. Participants could not explain why they chose these platforms, nor if they were effective in reaching their target audiences. It is clear that many of these organizations are not sure if their brand or messaging via social media is effective. There was almost no participation in online video sharing sites. Five participants noted that they had a presence on YouTube, with only one of the attendees (coincidentally a for-profit) utilizing Video. With web video growing exponentially each year, it is a great concern that so many of the attendees are not utilizing video to meet the branding needs of their firms. Mobile technology and smart phones are making the capture and upload of high definition video easier than ever before, and with web video driving more and more web traffic and dialog conducted on social media, it may be necessary to conduct research to determine why the use of web video may be lagging for these groups and perhaps for other organizations.

\section{Social media strategic plans}

Between the two groups five organizations reported the use of social media strategic plans that laid out clear goals and objectives. More than one organization suggested that they were using social media simply to utilize the tools. Upon reflection it is clear that these groups were unable to measure the success of their social media as well as determine their effectiveness. The for-profit participants seemed to agree that it would be in their best interests to identify goals and measurable objectives, thus beginning the process of data collection to measure their success. The non-profit participants appeared to struggle with this concept. This may be the result of the lack of resources within their organizations to conduct such efforts. Overall, organizations should create social media strategic plans to properly leverage these tools and foster connection and collaboration among their target audiences.

\section{Social media policies}

Between the two groups five organizations reported the existence of social media policies in their organization. This is an alarming observation. First, participants could not agree upon what constituted social media within their firms. Organizations must define social media. For example, is it Facebook and Twitter? What about future forms of social media; should they be factored in? Should video sharing sites be included as well? Also, organizations must clarify with their team members which information is the property of the individual versus the organization's as well as defining what constitutes confidential information. In addition, organizations must identify members of their teams who will monitor their social media and thus engage in it to facilitate dialog.

\section{Conclusion}

Both of the researchers were enlightened by the discussion and subsequent reflections. The organizations found great value in utilizing social media to encourage dialog, foster collaboration, and perhaps

*Corresponding author: Michelle Schmude, Department of Mass Communications, King's College, USA, Tel: 570-208-5947; E-mail: MichelleLawrence-Schmude@kings.edu Received October 10, 2013; Accepted October 11, 2013; Published October 12 2013

Citation: Schmude M, Weiland SJ (2013) Non-Profits Vs For-Profits in the Game of Social Media: Critical Observations and Reflections. J Mass Communicat Journalism 3: e143. doi:10.4172/2165-7912.1000e143

Copyright: () 2013 Schmude M. This is an open-access article distributed under the terms of the Creative Commons Attribution License, which permits unrestricted use, distribution, and reproduction in any medium, provided the original author and source are credited. 
Citation: Schmude M, Weiland SJ (2013) Non-Profits Vs For-Profits in the Game of Social Media: Critical Observations and Reflections. J Mass Communicat Journalism 3: e143. doi:10.4172/2165-7912.1000e143

Page 2 of 2

bolster their web traffic or even generate revenue. However, it did not matter if the participants in the session were in the business of making money or serving a philanthropic cause when it came to social media.
Participants must regroup and plan, arrive at a social media strategy with a strong social media policy as a backbone, and continue to execute their social media efforts with zeal. 